\title{
Effect of Specular Reflection on Out-of-Plane Ultrasonographic Images Reconstructed from Three-Dimensional Data Sets
}

\author{
Ronald O. Bude, MD, Theresa Tuthill, PhD
}

The effect of specular reflection on ultrasonographic images reconstructed out of plane to the plane of acquisition of a three-dimensional volumetric data set was studied using two in vitro phantoms that incorporated structures exhibiting specular reflection. The phantoms were scanned transversely (axially) to form three-dimensional data sets, with coronal cross-sectional images reconstructed perpendicular to the plane of acquisition of the data sets. Directly scanned, nonreconstructed coronal images of the phantoms also were obtained in the same planes and from the same areas as the reconstructed coronal images. The direct and reconstructed coronal images were compared. Owing to the inher- ent directionality of specular reflectors, the reconstructed coronal images differed from the directly scanned images in two ways, containing some hyperechoic regions that were not present at direct coronal scanning and failing to contain other hyperechoic areas that were present at direct coronal imaging. We conclude that sonographic images reformatted from volumetric data sets may have a different appearance than images scanned directly in the same plane, independent of other factors such as resolution. This should be taken into account when such reformatted images are interpreted. KEY WORDS: Three-dimensional ultrasonography; Artifact; Reflection, specular.

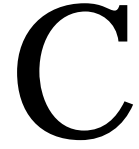

onstruction of reformatted planar ultrasound images from 3D data sets was described in the last decade.1,2 Initially, adoption of the technique was hampered by considerable technologic and computational requirements.

\section{ABBREVIATIONS}

3D, Three-dimensional

Received June 16, 1999, from the Department of Radiology, University of Michigan Medical Center, Ann Arbor, Michigan. Revised manuscript accepted for publication March 4, 2000.

Address correspondence and reprint requests to Ronald Bude, MD, Department of Radiology, B1D 502, University of Michigan Medical Center, 1500 East Medical Center Drive, Ann Arbor, MI 48109-0030.
Recently, however, the technology has improved considerably, so that many studies have evaluated the utility of this method, only a partial listing of which can be cited. ${ }^{3-19}$ Some potential advantages of this technique include (1) ability to visualize anatomic or pathologic features in optimum planes that cannot be insonated directly $2,3,5$; (2) improved evaluation of organs subject to respiratory motion, as the entire volume data set can be obtained during one breathhold ${ }^{18}$; (3) ability to perform the data acquisition portion of an ultrasonographic examination very quickly ${ }^{18}$; and (4) reduced operator dependence, which it is hoped will result in improved standardization and repeatability of examinations. 18

Specular reflectors are hyperechoic when insonated at, or near, perpendicularity, but they exhibit reduced, or absent, echogenicity at other insonation angles. Because of this directionality, we hypothesized that specular reflectors might produce artifacts in planar images reconstructed from 3D data sets, so that an 
image reconstructed in a plane other than the plane in which the 3D data set was obtained may have a different appearance than an image obtained by direct scanning in that plane (Fig. 1). Two in vitro models

Figure $1 A$ and $B$ are cubes that are exactly alike, composed of a sonolucent matrix containing hyperechoic central tubular inclusions. The solid arrows indicate the pulse propagation direction. The acoustic impedance of the tubular inclusions is different from that of the cube material so that the walls of the tubular inclusions are specular reflectors (the black curvilinear arcs at the tubular inclusions in $A$ and $B$ ). If cube $A$ is directly insonated from the side, the resultant slices in planes 1 and 2 should have the appearances indicated at $1^{*}$ and $2^{*}$, respectively (black margins of the tubular inclusion in $1^{*}$ are due to specular reflection; the thin hyperechoic area in $2^{*}$ results from the beam intersecting the edge of the tubular inclusion, where no specular reflection is present). If a $3 \mathrm{D}$ volumetric data set is obtained with the plane of acquisition orthogonal to the plane of reconstruction, as illustrated in cube $B$, the tubular inclusions should have a different appearance in images reconstructed in the same planes as planes 1 and 2. Cube $B$ is insonated with the pulse propagation direction perpendicular to its top, with the 3D volumetric data set obtained by translating the transducer across the top of the cube in the direction of the dashed arrow. With this scanning geometry, specular reflections occur at the top and bottom of the tubular inclusion in cube $B$. The planar image reconstructed at plane 3 (same plane as plane 1) should differ from that of plane 1 because the data set lacks the specular reflections present in direct scanning in plane 1 . The planar image reconstructed at plane 4 (same plane as plane 2) should differ from that of plane 2 as it contains the specular reflection produced when the pulse propagation direction is perpendicular to the top of cube $B$; no such specular reflection is present in plane 2 on direct scanning.

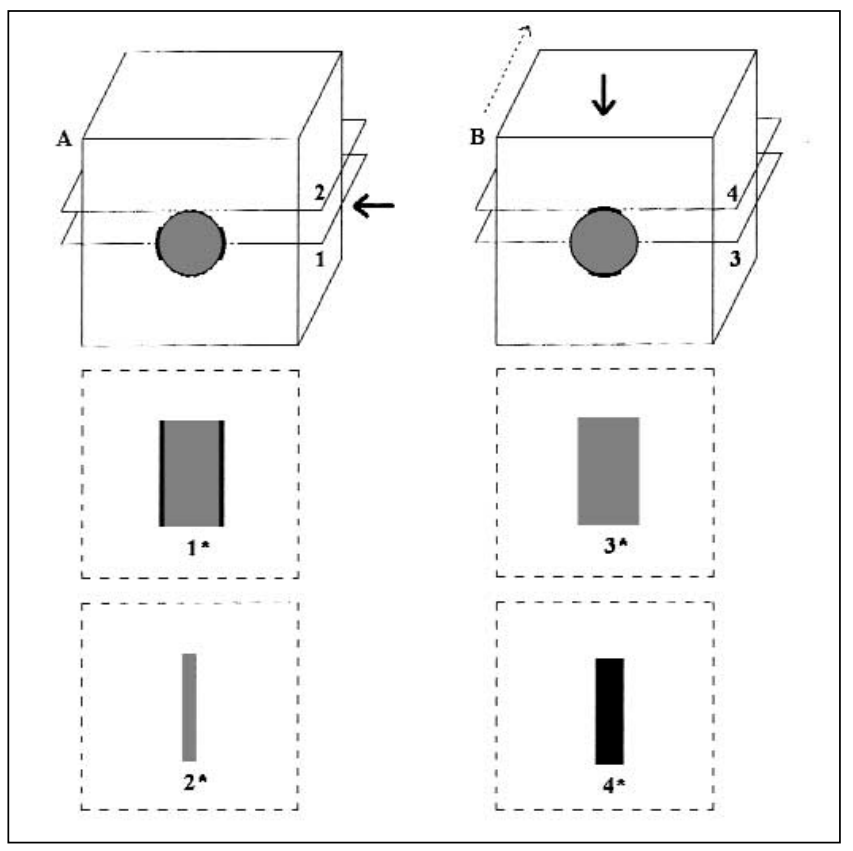

were constructed and scanned to evaluate our hypothesis. If it is correct, this effect of specular reflectors on planar images reconstructed from a 3D data set might lead to difficulty or confusion in interpretation of these images.

\section{MATERIALS AND METHODS}

$3 \mathrm{D}$ volume sets of sonographic data were obtained by scanning two ultrasound phantoms in the transverse plane. A commercially available ultrasound unit coupled to a position encoding device was used. Offline analysis was performed to reconstruct images of both phantoms in the coronal plane. Images of both phantoms were also obtained by direct scanning in the coronal plane. For each phantom the reconstructed coronal images were then compared to directly scanned coronal images to determine if specular reflectors produced discernible differences between the two sets of images. A detailed description of the experimental technique follows.

\section{Ultrasound Phantoms}

One phantom was constructed of long, linear hyperechoic scatterers, circular in cross section and approximately $3 \mathrm{~mm}$ in diameter. Scatterers that were circular in cross section were chosen so that transverse sections, obtained in any direction perpendicular to the long axis of the scatterer, would still be circular in cross section and have specular reflections at the near and far edges of the scatterers (Fig. 2). This phantom is subsequently referred to as the "parallel bundle phantom." The other phantom was constructed using a cadaver Achilles tendon to determine if the effects demonstrated in the parallel bundle phantom could be reproduced using a tissue model. This phantom is subsequently referred to as the "tendon phantom."

\section{Parallel Bundle Phantom}

The substrate in which the hyperechoic parallel bundles were suspended was prepared by dissolving unflavored gelatin in boiling water $(250 \mathrm{ml}$ water for every $20 \mathrm{~g}$ of gelatin). The phantom was formed in stages in a shallow plastic container. First, a base of gelatin approximately $2 \mathrm{~cm}$ thick was poured and cooled in a refrigerator until firm. Strips of gelatin of similar thickness, approximately $1.5 \mathrm{~cm}$ wide, were placed on top of the surface of the base layer to form a four-walled gelatin enclosure (approximately $2 \mathrm{~cm}$ high and $3 \times 7 \mathrm{~cm}$ on its sides) to hold the parallel bundles. Previous experience had shown us that 


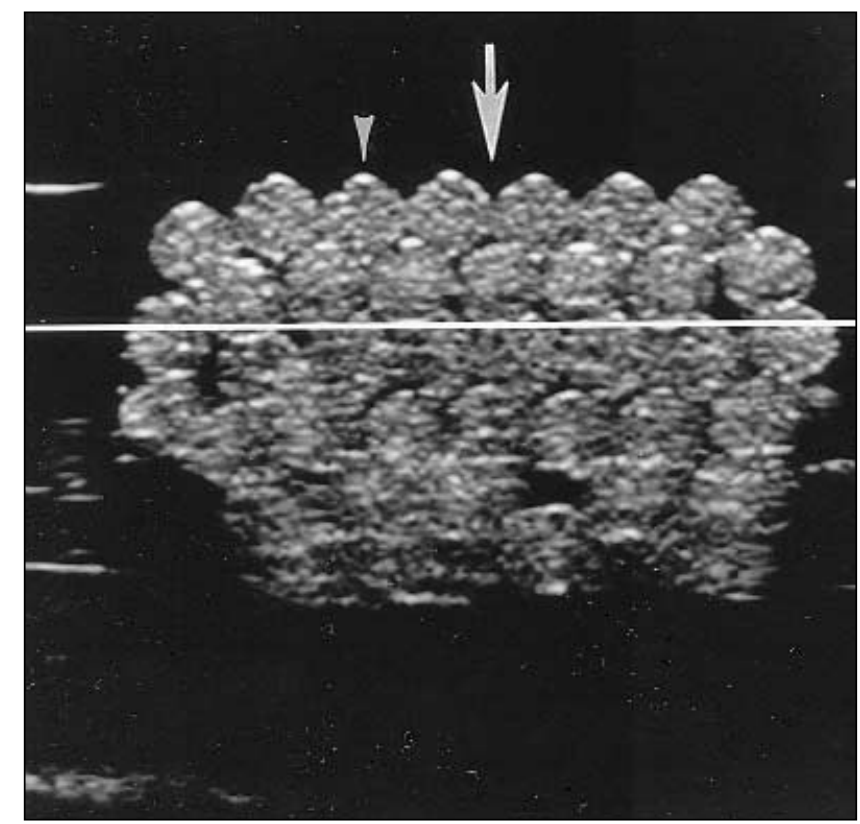

A

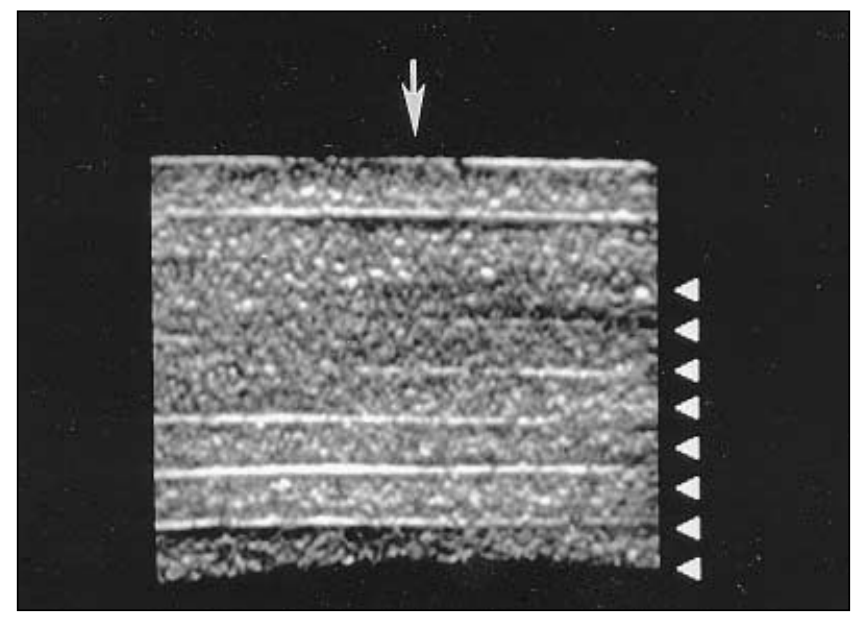

C

cooked spaghetti when insonated has a hyperechoic appearance that simulates the speckle of homogeneous organs such as the thyroid or testis, and that if spaghetti is suspended in gelatin it exhibits specular reflections at its near and far edges (even when it is stacked on itself). Thus, spaghetti was chosen as the hyperechoic scatterer for the phantom. An adequate amount of \#8 size spaghetti was cooked until tender, as if for eating. Once cooked, each strand of spaghetti measured approximately $3 \mathrm{~mm}$ in diameter. Lengths of spaghetti were carefully arranged parallel to each other in the gelatin well so that their sides touched. Layers of spaghetti were stacked to form the phantom. Constructed this way, the phantom consisted of layers of hyperechoic interfaces

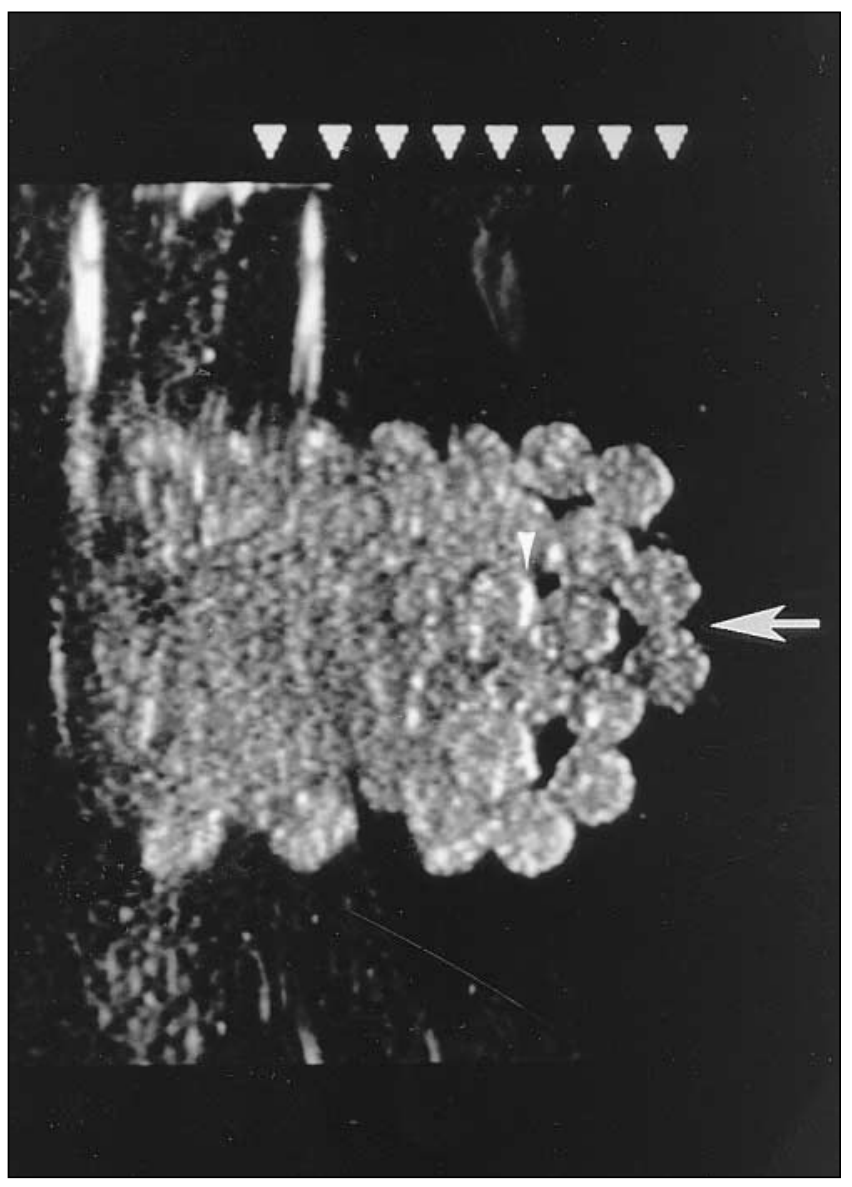

B

Figure 2 Ultrasonograms produced by direct scanning of the parallel bundle phantom. A, Direction of pulse propagation (arrow, single arrowhead) perpendicular to the top of the phantom, resulting in a transverse section. B, Pulse propagation perpendicular to the side of the phantom, resulting in a transverse section scanned in a different orientation than in A. C, Pulse propagation perpendicular to the side of the phantom but in coronal section. Note that the slightly curvilinear hyperechoic arcs (arrowheads) at the near and far edges of the bundles, due to specular reflection, are positioned in different locations in $\mathbf{A}$ than in B. (Ignore the white line in $\mathbf{A}$, which is a reference line for a coronal reconstruction not used in this figure.) Also note that the specular reflections in $\mathbf{C}$ are thin hyperechoic lines, which were never thicker than those illustrated in any coronal plane.

oriented parallel to each other (Fig. 2). Additional gelatin was then carefully poured over the spaghetti, taking care to eliminate all air from around the spaghetti, and cooled until set. Once formed, the phantom had the appearance of a solid block of gelatin with a parallel bundle of spaghetti suspended within it. 


\section{Tendon Phantom}

This phantom was composed of an approximately $7 \mathrm{~cm}$ length of cadaver Achilles tendon suspended in gelatin.

\section{Scanning Technique}

\section{Gray Scale Sonography}

Sonography was performed with a LOGIC $700 \mathrm{MR}$ ultrasound unit (GE Medical Systems, Milwaukee, WI) using an M12L variable frequency linear array transducer (which produces parallel image lines) operating at $13 \mathrm{MHz}$, with multiple focal zones at the region of interest. The following settings were used for the tendon phantom: dynamic range, 78; edge enhancement, 4; map, $E^{*}$ (linear mapping); frame averaging, 0 . The following settings were used for the parallel bundle phantom: dynamic range, 69; edge enhancement, 6; map, E; frame averaging, 0 . For each phantom, the same settings were used for both the 3D volume acquisition and the direct scans obtained for comparison with reconstructed images.

\section{$3 D$ Volume Data Acquisitions}

$3 \mathrm{D}$ volume data acquisitions were obtained by imaging according to the following technique, described elsewhere. ${ }^{20}$ The transducer is affixed to a freehand scan system in which the B-scan plane is perpendicular to the scanning motion. A framework allows the transducer to slide along a track but prevents lateral, range, and rotational movement. A computer-controlled position encoder records the transducer location, and B-scans are acquired at fixed intervals of 100 $\pm 10 \mu \mathrm{m}$. It took approximately 5 min per 3D volume acquisition to scan and obtain the data. Each B-scan was digitized as $379 \times 380$ pixel images, with each pixel corresponding to a $100 \mu \mathrm{m} \times 100 \mu \mathrm{m}$ region. Once the data sets were obtained for both phantoms, offline coronal images orthogonal to the plane of original image acquisition were obtained using MATLAB (Mathworks, Natick, MA) image processing software. By averaging in the original axial dimension, the desired reconstruction slice thickness was obtained. Calibration experiments on a homogeneous speckle phantom have previously shown the slice thickness of the transducer, operated under the chosen parameters, to be approximately $200 \mu \mathrm{m}$. Images were therefore reconstructed from the 3D data set at $200 \mu \mathrm{m}$ thicknesses, throughout the entire thickness of the phantoms, to allow accurate comparison to directly scanned images. The images that best showed the discrepancy between direct coronal and reconstructed coronal images were chosen for illustration.

\section{Direct Coronal Scanning}

Direct scans in the coronal plane of both the parallel bundle phantom and the tendon phantom were obtained to compare with the scans reconstructed in the coronal plane from the $3 \mathrm{D}$ volume data sets.

\section{RESULTS}

\section{Parallel Bundle Phantom}

Figure 2 shows representative images from direct transverse scanning (pulse propagation direction perpendicular to the top [Fig. 2A] and to a side [Fig. 2B]) and coronal scanning (Fig. 2C). Thin linear hyperechoic areas are present at the near and far walls of the bundles due to specular reflections.

Representative coronal images derived from the 3D data set are illustrated in Figure 3. Note that the hyperechoic bands in the reconstructed images (Fig. 3B, C) are broader than those in the directly scanned coronal image (Fig. 2C), which were very thin and never had an appearance thicker than that illustrated on any of the images obtained. Although broad hyperechoic bands similar to those in Figure 3B,C were not present in every $200 \mu \mathrm{m}$ thick reconstructed image throughout the entire thickness of the phantom, they were present when the reconstruction plane intersected a specular reflector. These broad hyperechoic bands are a major difference between the longitudinal images reconstructed in the coronal plane and the longitudinal images from direct scanning in the coronal plane. Conversely, coronal images reconstructed from the same data set but with the reconstruction plane at a level that does not include specular reflections showed a virtually complete absence of linear hyperechoic regions (Fig. 3D). This contrasts with the direct coronal scan perpendicular to the near and far walls of the bundles (Fig. 2C), which shows multiple linear hyperechoic regions produced by specular reflections.

\section{Tendon Phantom}

Representative images from direct transverse and coronal scanning are illustrated in Figure 4. Multiple, linear, parallel hyperechoic interfaces that are very thin are present in the longitudinal coronal image (Fig. 4C). Linear hyperechoic bands thicker than those illustrated could not be elicited by direct coronal scanning. As with the parallel bundle phantom, coronal images of the tendon reconstructed from the 
3D data set show broad, linear areas of increased echogenicity (Fig. 5B), which were much broader than any present at direct coronal scanning (Fig. 4C). Additionally, sharply defined, thin, parallel linear hyperechoic regions were not as prevalent in the reconstructed coronal images as they were in the images obtained from direct coronal scanning (compare Fig. 5B with Fig. 4C).

Figure 3 Reference transverse slice (A) from the 3D volumetric data set obtained with pulse propagation perpendicular to the top of the phantom and coronal images (B-D) of the parallel bundle phantom reconstructed from the $3 \mathrm{D}$ data set. White line spanning the image from side to side and intersecting the parallel bundles in A denotes the level of reconstruction of $\mathbf{B}$. Note the five thick hyperechoic bands in B. These are produced by the reconstruction plane in $\mathbf{A}$ intersecting and including the locations of specular reflections (which are obscured by the white line in A). Hyperechoic bands this thick could not be produced by direct coronal imaging. (In this and all other coronal reconstructions, the top of the reconstructed coronal image corresponds to the right side of the reference transverse image.) Coronal reconstruction in $\mathbf{C}$ shows the widest band of increased echogenicity that could be produced in the coronal reconstruction plane. Coronal reconstruction in D was performed through the middle of the bundles, where no specular reflections were present in the 3D data set (except for the area at the bottom left of the image, which included the edge of one of the spaghetti strands). Thus, the image lacks the long white lines at the margins of the spaghetti bundles that were present at direct coronal scanning (Fig. 2C).

\section{DISCUSSION}

To test our hypothesis that specular reflectors cause images reconstructed from a $3 \mathrm{D}$ volumetric data set to potentially have a different appearance from images obtained in the same plane by direct scanning, we constructed and studied two phantoms. One, the parallel bundle phantom, exhibited symmetry to specular reflection (i.e., whether it was scanned from the top or the side, the specular reflections were located at the near and far walls of

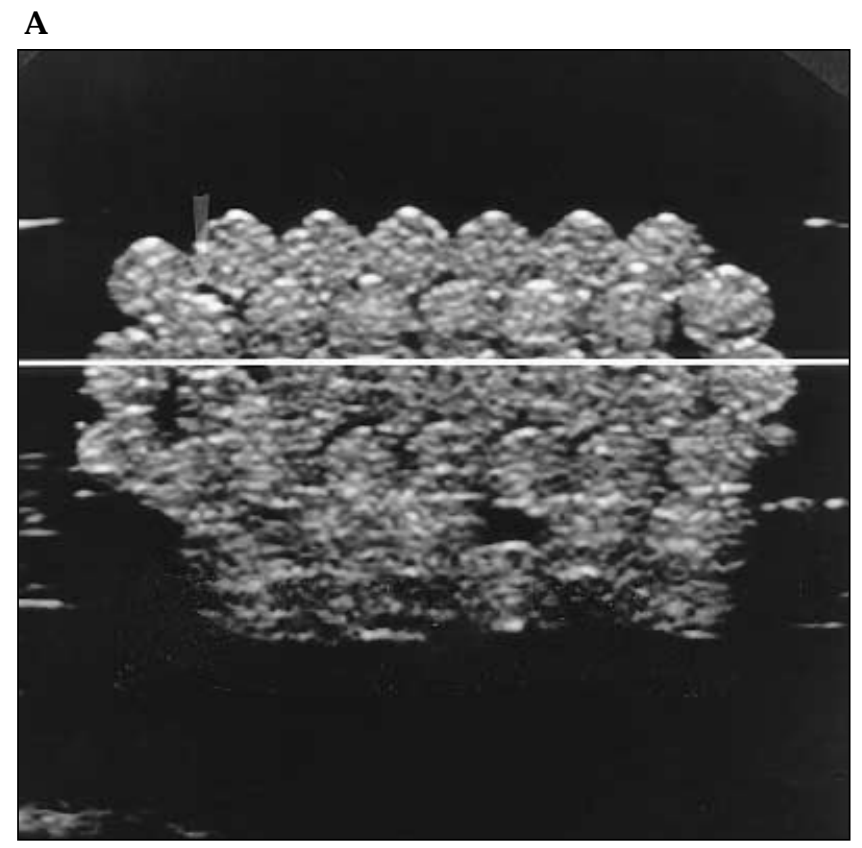

B

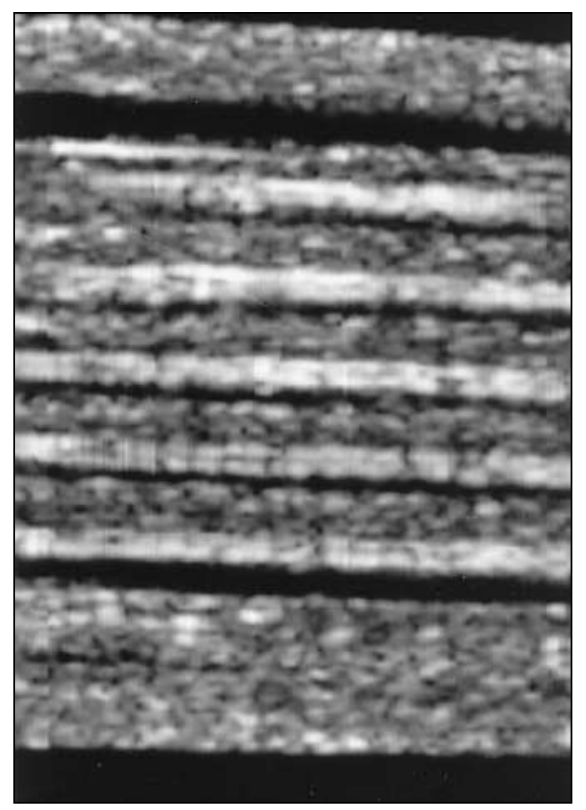

C

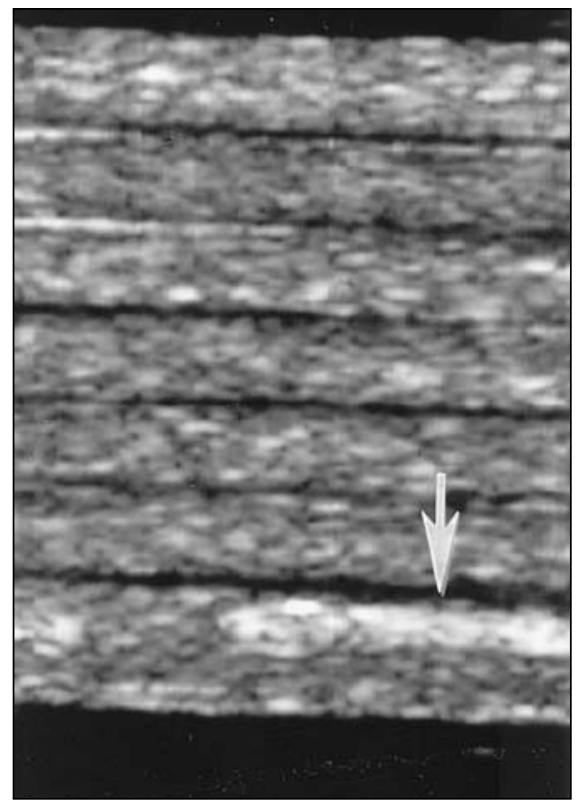

D

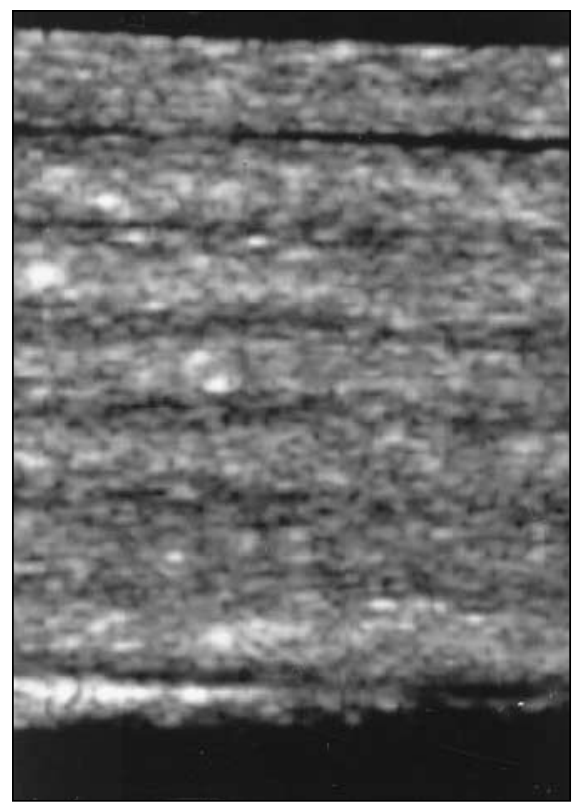


the structures producing the specular reflections [Fig. 2A, B]). The other phantom, the tendon phantom, was studied to show that the suspected effects of specular reflection, once demonstrated in the first phantom, could be produced from tissue as well. The Achilles tendon was used because it is the largest tendon in the body; however, we expect that similar results would be obtained from any tendon. A phantom incorporating an Achilles tendon, rather than direct scans of in vivo Achilles tendon, was used for two reasons. First, the medial and lateral skin margins of the posterior heel are curved. This interfered with obtaining direct coronal images at normal incidence to the long axis of the tendon, even using a stand-off pad. Since tendons are highly anisotropic (the specular reflectors well seen at normal incidence quickly disappear when scanning deviates more than just a few degrees from

Figure 4 Ultrasonograms produced by direct scanning of the tendon phantom (arrows denote the direction of pulse propagation). $\mathbf{A}$ and $\mathbf{B}$ are transverse images and $\mathbf{C}$ is a coronal image (these images were obtained in the same planes as A, B, and C of Fig. 2, respectively). In A, the direction of pulse propagation is perpendicular to the top of the tendon, whereas in $\mathbf{B}$ the direction of pulse propagation is perpendicular to the side of the tendon (arrows). Note in $\mathbf{A}$ the multiple short, linear hyperechoic bands, which are specular reflections, oriented perpendicular to the image lines. In B, similar specular reflections are present again perpendicular to the image lines, but they are now oriented perpendicular to those in A. (Hyperechoic strands projecting from the tendon margins are thin strands of residual connective tissue adhering to the tendon.). The same effect was seen throughout the entire tendon. In the coronal image (C), note the thin, lengthwise linear hyperechoic strands produced as the sound wave encounters the specular reflectors of $\mathbf{B}$. Throughout the entire tendon, none of the linear hyperechoic strands were thicker than those illustrated.

B

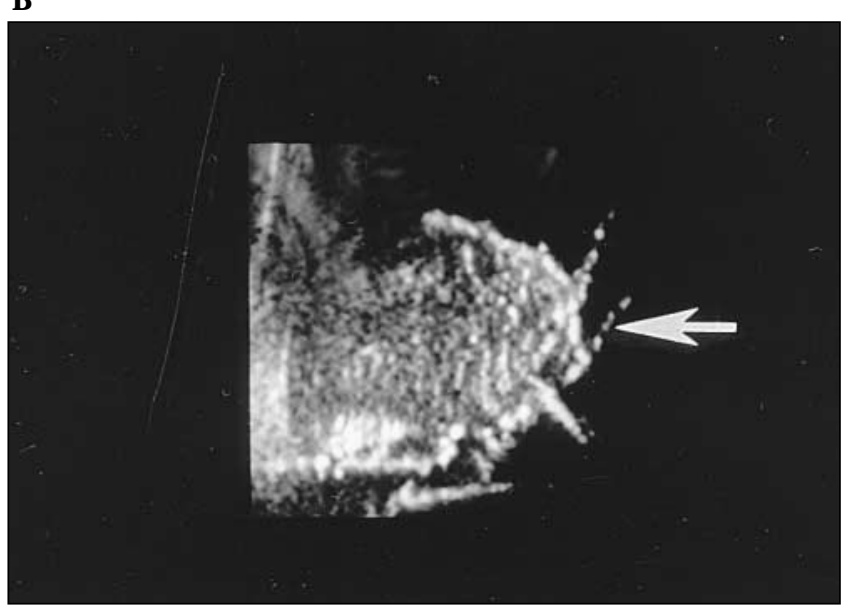

normal incidence), it was important for us to scan perpendicular to the tendon. The in vitro model allowed this owing to its flat sides (the tendon was positioned in the gelatin with its long axis parallel to the sides of the phantom). Second, this method prevented patient motion and avoided corruption of the data set.

Scanning the parallel bundle phantom produced the results we expected. First, images reconstructed in the coronal plane, perpendicular to the transverse plane of data set acquisition, at times generated wide, linear hyperechoic regions (Fig. 3B, C) that are an artifact of the technique and are not present with direct coronal scanning (Fig. 2C). We believe this is due to the fact that the plane of the reconstructed scan is parallel to, with its thickness encompassing, a broad area of specular reflection (Fig. 1), which is then included in the reconstructed image as a broad area of

A

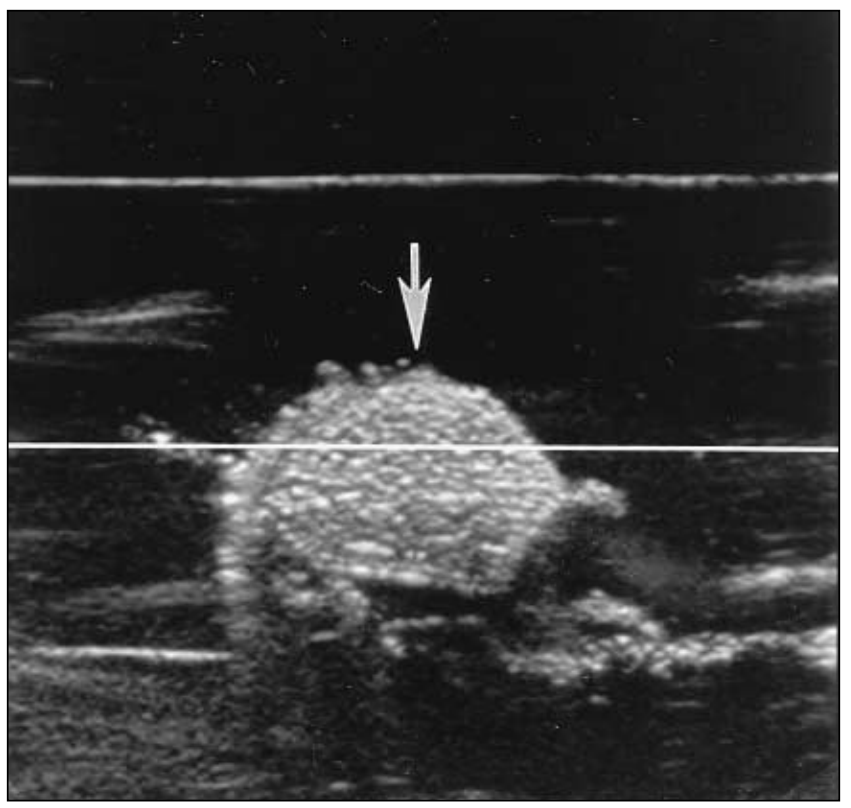

C

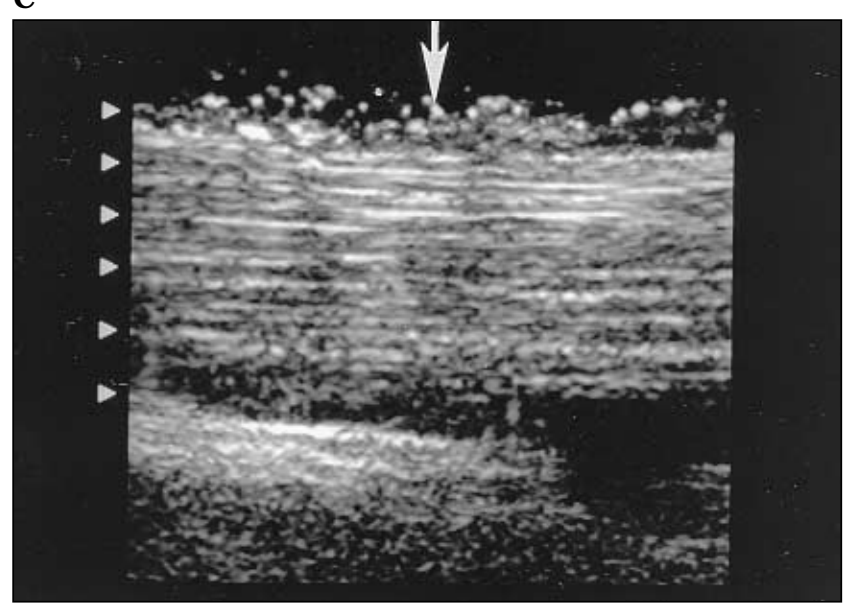


echogenicity. Second, coronal images reconstructed at a level that does not include the specular reflections produced by transverse scanning perpendicular to the plane of coronal reconstruction do not exhibit the same linear hyperechoic regions at the edges of the bundles (Fig. 3D) that are present with direct coronal scanning (Fig. 2C). We believe this is because the 3D data set, since it is scanned in a different plane than the plane of reconstruction, does not have specular reflectors in the same locations where they are present on direct coronal scanning. Thus, not only may reformatted images contain artifactual information (the broad hyperechoic bands) but in addition they may fail to contain information (linear specular reflective interfaces) that is present at direct scanning.

After the parallel bundle phantom was studied, we sought to determine if the same effects could be observed with biologic tissue. Tendon was chosen as the tissue of interest because it contains a large number of specular reflectors. Our results show that the same effects observed in the parallel bundle phantom are produced in tendon as well. Thick hyperechoic lines (Fig. 5B), caused by inclusion of specular reflections, are present in the reconstructed coronal images that were not present in the directly scanned coronal images (Fig. 4C). Furthermore, fewer thin hyperechoic interfaces were present in the reconstructed coronal images than in the directly scanned coronal images.

It should be noted that our experiment was designed to show the maximum effect of the artifactual appearance produced by the inclusion of specular reflections in images reconstructed out of plane to the plane of data acquisition. The hyperechoic bands produced by inclusion of specular reflections in the reconstructed images should become thinner as the reconstructed scan plane approaches the plane of data set acquisition.

One limitation of our study is that tendon exhibits more specular reflections than many other organs do. Therefore, results in some other organs may not be as prominent as those observed in the tendon we studied. Nonetheless, our results have demonstrated

Figure 5 Reference transverse image (A) from a 3D volumetric data set obtained with pulse propagation in the direction of the arrow and longitudinal coronal image (B) of the tendon reconstructed from the data set (white line in A denotes the level of the coronal reconstruction in B). In B, note two things: first, a broad hyperechoic band is present at the top of the reconstructed image (arrow) produced by inclusion of a specular reflector in the reconstructed image (which was visible in the reference transverse view but is obscured by the white reference line in A). Bands this wide could not be produced at direct coronal scanning (Fig 4C). Second, note that this reconstructed coronal image contains fewer sharply defined, thin, linear hyperechoic strands than are present in Figure 4C. This finding was noted throughout the series of reconstructed coronal images.

A

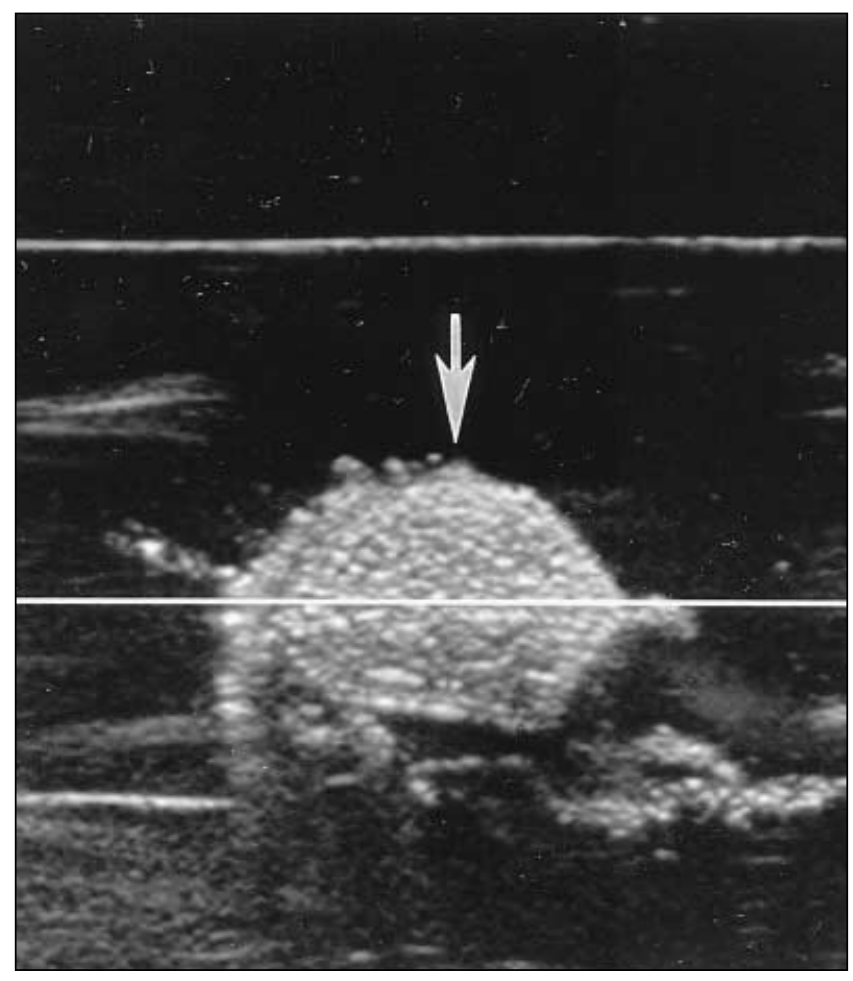

B

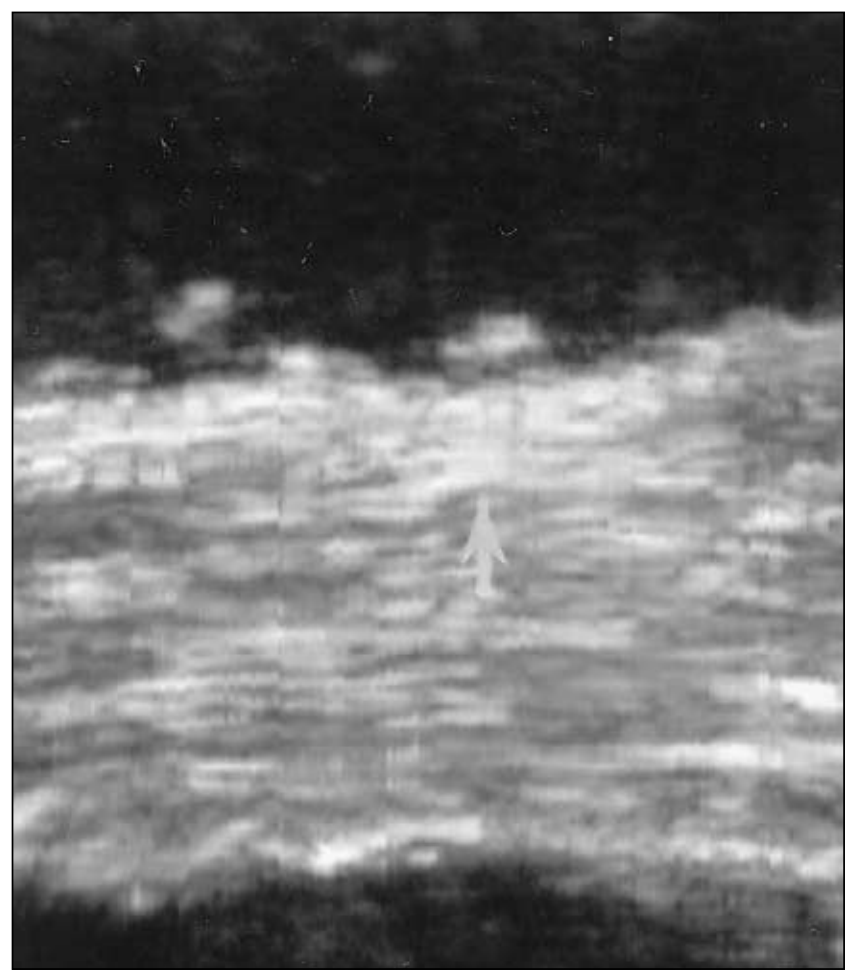


the effects of specular reflections on reconstructed images, and these effects should come into play in any organ or structure that exhibits specular reflections. Furthermore, tendon sonography is potentially a fruitful area for the application of reformatted outof-plane imaging. In the rotator cuff, where the axial and sagittal images that are usually obtained are unfamiliar and confusing to the referring surgeon, coronal reformatted images may help to convey better the full extent of a tendon tear. Thus, in this application alone, it may be important to understand the appearance of the artifacts that our study has demonstrated.

In conclusion, specular reflections have a different appearance in images reconstructed from a 3D data set than they do in directly scanned images. This may cause a potentially confusing appearance in the reconstructed images. In clinical images, the result of this effect may range from insignificant in those organs in which specular reflections play a minor role in image formation to potentially problematic in those organs whose appearance depends more strongly on specular reflection. In problematic cases, it may be necessary to perform direct scanning in the same plane as the reconstructed image to resolve the confusion. Further study is required to determine the extent and overall importance of these artifacts.

\section{REFERENCES}

1. Raichlen JS, Trivedi SS, Herman GT, et al: Dynamic threedimensional reconstruction of the left ventricle from twodimensional echocardiograms. J Am Coll Cardiol 8:364, 1986

2. Halliwell M, Key H, Jenkins D, et al: New scans from old: Digital reformatting of ultrasound images. Br J Radiol 62:824, 1989

3. Pretorius DH, Nelson TR, Jaffe JS: 3-dimensional sonographic analysis based on color flow Doppler and gray scale image data: A preliminary report. J Ultrasound Med 11:225, 1992

4. Pretorius DH, Nelson TR: Prenatal visualization of cranial sutures and fontanelles with three-dimensional ultrasonography. J Ultrasound Med 13:871, 1994

5. Hamper UM, Trapanotto V, Sheth S, at al: Three-dimensional US: Preliminary clinical experience. Radiology 191:397, 1994

6. Steiner H, Staudach A, Spitzer D, et al: Three-dimensional ultrasound in obstetrics and gynaecology: Technique, possibilities and limitations. Hum Reprod 9:1773, 1994

7. Pretorius DH, Nelson TR: Fetal face visualization using three-dimensional ultrasonography. J Ultrasound Med 14:349, 1995

8. Downey DB, Fenster A: Vascular imaging with a threedimensional power Doppler system. AJR 165:665, 1995
9. Pretorius DH, House M, Nelson TR, et al: Evaluation of normal and abnormal lips in fetuses: Comparison between three- and two-dimensional sonography. AJR 165:1233, 1995

10. Bonilla-Musoles F, Raga F, Osborne NG, et al: Use of three-dimensional ultrasonography for the study of normal and pathologic morphology of the human embryo and fetus: Preliminary report. J Ultrasound Med 14:757, 1995

11. Riccabona M, Nelson TR, Pretorius DH, et al: Distance and volume measurement using three-dimensional ultrasonography. J Ultrasound Med 14:881, 1995

12. Elliot TL, Downey DB, Tong S, et al: Accuracy of prostate volume measurements in vitro using three-dimensional ultrasound. Acad Radiol 3:401, 1996

13. Capineri L, Masotti L, Rocchi S, et al: Nearly real-time visualization of arbitrary two-dimensional sections from three-dimensional acquisition. Ultrasound Med Biol 22:319, 1996

14. Riccabona M, Johnson D, Pretorius DH, et al: Three dimensional ultrasound: Display modalities in the fetal spine and thorax. Eur J Radiol 22:141, 1996

15. Chan L, Lin WM, Uerpairojkit B, et al: Evaluation of adnexal masses using three-dimensional ultrasonographic technology: Preliminary report. J Ultrasound Med 16:349, 1997

16. Sivan E, Chan L, Uerpairojkit B, et al: Growth of the fetal forehead and normative dimensions developed by threedimensional ultrasonographic technology. J Ultrasound Med 16:401, 1997

17. Wu MH, Hsu CC, Huang KE: Detection of congenital mullerian duct anomalies using three-dimensional ultrasound. J Clin Ultrasound 25:487, 1997

18. Rankin RN, Fenster A, Downey DB, et al: Three-dimensional sonographic reconstruction: Techniques and diagnostic applications. AJR 161:695, 1993

19. Tuthill TA, Krucker JF, Fowlkes JB, et al: Automated three-dimensional US frame positioning computed from elevational speckle decorrelation. Radiology 209:575, 1998

20. Fenn RC, Fowlkes JB, Moskalik A, et al: Acoustical Imaging. New York, Plenum, 1997, p 237 